Pacific Journal of Mathematics

RECURSIVELY ENUMERABLE SETS AND VAN DER
WAERDEN'S THEOREM ON ARITHMETIC PROGRESSION 


\title{
RECURSIVELY ENUMERABLE SETS AND VAN DER WAERDEN'S THEOREM ON ARITHMETIC PROGRESSIONS
}

\author{
CARl G. Jockusch, JR. and IraJ Kalantari
}

\begin{abstract}
Subsets of the set $\omega$ of nonnegative integers which possess some algebraic structure are interesting since they are most likely to give number-theoretic information. Arithmetic progressions are one of the simplest structures to observe. Effectiveness of any kind of information is of course an important factor. It seems that a study of possible interrelationships between combinatoric and number-theoretic properties of recursively enumerable (r.e.) subsets of $\omega$ might be interesting. In this paper we study van der Waerden's theorem on arithmetic progressions in this light.
\end{abstract}

1. Introduction. Schur, while working on the distribution of quadratic residues in $\mathbf{Z}_{p}$, had conjectured that: if $\omega$ is split into two disjoint sets, then an arithmetic progression of any desired length may be found in at least one of the sets (see [1].)

Baudet, a student at Göttingen, had mentioned this conjecture to van der Waerden. In 1927, van der Waerden published an elementary but insightful proof of the conjecture to which he referred as Baudet's conjecture (see [5].)

Van der Waerden [6] gives an interesting account of how in an afternoon of 1926, he, Artin and Schreier discussed the conjecture and found a proof for it. In [5] (and [6]), van der Waerden gives a proof of a more general form of the conjecture: if $\omega$ is equal to the disjoint union of the sets $A_{1}, \ldots, A_{n}$, then given an arbitrary $k$, at least one $A_{l}$ contains an arithmetic progression of length $k$. Moreover, for a proof he demonstrated the existence of a function $f$ such that given $n$ (the number of cells in the partition) and $k$ (the desired length of arithmetic progression), then a mere partitioning of $\{0,1, \ldots, f(n, k)\}$ into $n$ cells in any manner, yields an arithmetical progression of length $k$ in at least one of the cells. Furthermore, the given function $f$ is indeed a recursive one. For our purposes we state

VAN DER WAerden's TheOREM. Let $A \subset \omega$. Pick $k$. Then either $A$ or $\omega-A$ has an arithmetic progression of length $k$. 
From a recursion theoretic point of view it is reasonable to ask whether van der Waerden's theorem holds when only r.e. sets are considered. This question may not at first appear to make sense because $\omega-A$ need not be r.e. even when $A$ is r.e. To remedy this difficulty, we let the role of $\omega-A$ be taken over by arbitrary r.e. sets disjoint from $A$. To avoid trivialities we also require that a set have infinitely many pairwise disjoint arithmetic progressions of length $k$ rather than just a single such progression. In $\$ 3$ we use the finite form of van der Waerden's theorem mentioned above to show that for any r.e. set $A$, either $A$ contains arbitrarily long arithmetic progressions or for each $k$ there is an r.e. set $B_{k}$ disjoint from $A$ which contains infinitely many pairwise disjoint arithemtic progressions of length $k$. One might hope that this result could be strengthened to show that for any r.e. set $A$, either $A$ or some r.e. set disjoint from $A$ contains arbitrarily long arithmetic progressions. Our main result, proved in $\$ 4$ using the priority method, refutes this conjecture by giving an example of an r.e. set which contains no arithmetic progression of length three and yet intersects every r.e. set which contains arbitrarily long arithmetic progressions. Section 5 gives a corollary based on Szemerédi's generalization of van der Waerden's theorem. Section 2 contains the key definitions and $\$ 6$ generalizes our main result. Finally some open questions are listed in $\$ 7$.

2. Definitions and some notation. By the length of an arithmetic progression we mean the number of the terms in it. Let $A \subset \omega$.

Definition 1. $A$ is thick if $A$ contains arithmetic progressions of any length $k \in \omega$.

Definition 2. Let $k \in \omega . A$ is $k$-thin if $A$ does not contain an arithmetic progression of length $k$. (This notion will also be used for subsets of rationals.)

Definition 3. Let $k \in \omega$. $A$ is $k$-thick if there is no finite set $F \subset \omega$ such that $A-F$ is $k$-thin.

Definition 4. $A$ is thin if $A$ is $k$-thin for some $k \in \omega$.

Some trivial observations about these notions will be useful:

(a) $A$ is thick iff $A$ is $k$-thick for all $k \in \omega$.

(b) $A$ is thick iff $A$ is not $k$-thin for any $k \in \omega$.

If $a, b \in \omega$, then $(a, b]$ denotes the set of elements of $\omega$ between $a$ and $b$ together with $b$. The set of rational numbers is denoted by $\mathbf{Q}$. For 
any finite sequence $q_{1}, \ldots, q_{n}$ of rational numbers define

$$
\Delta q_{i}=q_{i+1}-q_{i} \text { for } 1 \leq i<n
$$

and

$$
\Delta^{2} q_{l}=\Delta q_{i+1}-\Delta q_{i}=q_{i+2}-2 q_{i+1}+q_{i} \text { for } 1 \leq i<n-1 .
$$

These are the usual first and second difference operators. Note that $\Delta$ and $\Delta^{2}$ are linear in the obvious sense. $\mathscr{P}(\mathbf{Q})$ denotes the collection of all subsets of $\mathbf{Q}$ while $\mathscr{P}_{\text {fin }}(\mathbf{Q})$ denotes the collection of all finite subsets of $\mathbf{Q}$. For $C \subseteq \mathbf{Q}$, define the operator

$$
\Phi_{C}: \mathscr{P}(\mathbf{Q}) \rightarrow \mathscr{P}(\mathbf{Q})
$$

by

$$
\Phi_{C}(B)=\left\{\sum_{i=1}^{n} c_{i} b_{i} \mid c_{i} \in C \text { and } b_{1}, \ldots, b_{n} \text { are distinct elements of } B\right\} .
$$

$\Phi_{C}$ is finitely based if $C$ is finite. Note that finitely based operators map finite sets to finite sets.

Finally, $W_{e}$ denotes the $e$ th r.e. subset of $\omega$ in an acceptable enumeration of all such sets. For all other background on recursion theory, we refer the reader to Rogers [3].

\section{The positive direction.}

THEOREM 5. If $A$ is r.e. and not thick, then for each $k$ there is an r.e., $k$-thick set $B_{k}$ which is disjoint from $A$.

Proof. Suppose $A$ is $n$-thin. It suffices to construct $B_{k}$ for $k>n$. Fix $k>n$. By van der Waerden's theorem, there is a bound $b_{k}$ such that whenever $\left(0, b_{k}\right]$ is partitioned into two sets, one of the sets is not $k$-thin. For each $i$, let

$$
F_{i}=\left(b_{k} i, b_{k}(i+1)\right] \text {. }
$$

So $F_{0}, F_{1}, \ldots$ are pairwise disjoint translates of $F_{0}=\left(0, b_{k}\right]$. Thus whenever any $F_{i}$ is partitioned into two sets, one of the sets is not $k$-thin.

Let

$$
\begin{aligned}
& m_{k}=\text { the largest number } m \text { such that } \\
& \qquad\left|A \cap F_{i}\right|=m \text { for infinitely many } i .
\end{aligned}
$$

Note that $m_{k}$ exists because $\left|A \cap F_{l}\right| \leq\left|F_{i}\right|=b_{k}$ for all $i$. 
Choose $i_{k}$ such that

$$
i \geq i_{k} \Rightarrow\left|A \cap F_{l}\right| \leq m_{k}
$$

(Of course $m_{k}$ and $i_{k}$ are not obtained uniformly in $k$, but this is no obstacle since we are not claiming that $B_{k}$ is r.e. uniformly in $k$.)

Let $A^{s}$ be a recursive enumeration of $A$ and define

$$
B_{k}=\left\{b \mid \exists s\left(\exists i \geq i_{k}\right)\left[\left|A^{s} \cap F_{i}\right|=m_{k} \& b \in F_{l}-A^{s}\right]\right\} .
$$

Clearly, $B_{k}$ is r.e. Also, $B_{k} \cap A=\varnothing$ since otherwise $\left|A \cap F_{i}\right|>m_{k}$ for some $i \geq i_{k}$ contrary to the choice of $i_{k}$.

To show that $B_{k}$ is $k$-thick, it suffices to show that $B_{k} \cap F_{i}$ is not $k$-thin whenever $\left|A \cap F_{i}\right|=m_{k}$ and $i \geq i_{k}$, since there are infinitely many such $i$ by the choice of $m_{k}$. To see this, let $\left|A \cap F_{l}\right|=m_{k}$ and $i \geq i_{k}$. Then $A \cap F_{i}$ and $B_{k} \cap F_{i}$ partition $F_{i}$ into two sets, one of which is not $k$-thin by the choice of $b_{k}$. However, $A$ is $n$-thin, $k>n$, and $A \cap F_{l} \subseteq A$; hence $A \cap F_{i}$ is $k$-thin. Therefore $B_{k} \cap F_{l}$ is not $k$-thin.

We observe an interesting corollary. Recall $A$ is simple if $A$ is r.e. and coinfinite, but it meets every infinite r.e. set.

COROLlaRY 6. Every simple set is thick.

Corollary 6 may also be proved easily without using van der Waerden's theorem. The observation needed is that for any recursive sequence $\left\{F_{l}\right\}$ of pairwise disjoint finite sets of uniformly bounded cardinality and any simple set $A, F_{i}$ is a subset of $A$ for infinitely many $i$. (See [2], pp. 116-117.)

4. The negative direction. We now prove our main result using a priority construction. The construction is a finite injury one in the sense that each positive requirement contributes at most one element to the r.e. set $A$ being constructed. However, it is a bit unusual because an individual negative requirement may (permanently) restrain infinitely many numbers from $A$. The positive requirements are satisfied nonetheless because any finite set of negative requirements together restrain only a thin set of numbers from $A$.

THEOREM 7. There is a 3-thin r.e. set A which intersects every thick r.e. set. 
Proof. It is necessary and sufficient to satisfy the following requirements in the construction of the r.e. set $A$ :

$$
\begin{array}{ll}
N_{-1}: & A \text { is } 3 \text {-thin; } \\
P_{e}: & W_{e} \text { is thick } \rightarrow A \cap W_{e} \neq \varnothing .
\end{array}
$$

To satisfy $N_{-1}$, it suffices to keep out of $A$, with highest priority, any numbers which form an arithmetic progression of length 3 with numbers already in $A$ (and add at most one new element to $A$ at a time).

Let

$$
A^{*}=\{n \mid A \cup\{n\} \text { is not 3-thin }\} .
$$

Hence, to satisfy $N_{-1}$ we must arrange $A \cap A^{*}=\varnothing$. Now since $A$ is r.e., $A^{*}$ is also an r.e. set; say $A^{*}=W_{j}$. Clearly, to satisfy $P_{j}$ it is necessary that $A^{*}=W$, be $k$-thin for some $k$. The following proposition shows that we may not choose $k=3$.

Proposition 8. If $A$ is r.e. and 3-thin and $A$ intersects every thick r.e. set, then $A^{*}$ is 3-thick.

Proof. Clearly $A$ has to be infinite. Suppose $a, b \in A, a<b \leq 2 a$ and $b-a$ is even. Then the numbers $2 b-a,(a+b) / 2$ and $2 a-b$ are all in $A^{*}$ and form an arithmetic progression of length 3 . Thus if $A^{*}$ is not 3-thick, there are only finitely many pairs $(a, b)$ as above. But then

$$
W=\{b \mid(\exists a \in A)[a<b \leq 2 a \& b-a \text { is even }]\}
$$

is a thick r.e. set having only finite intersection with $A$. This is a contradiction. Hence $A^{*}$ is 3 -thick.

In view of the above proposition it is reasonable to ask whether, under the same hypothesis, it can be shown that $A^{*}$ is thick (which would refute the theorem we are trying to prove). The answer is no. The explanation lies in the fact that every element of $A^{*}$ may be written as $c_{1} a_{1}+c_{2} a_{2}$ where $a_{1}$ and $a_{2}$ are distinct elements of $A$ and $c_{1}$ and $c_{2}$ are elements of the fixed finite set $C=\left\{-1, \frac{1}{2}, 2\right\}$. The arithmetic progressions of length 3 in $A^{*}$ arise only because $C$ contains an arithmetic progression of length 3 . On the other hand, if we consider the fact that every element of $A^{*}$ may be written as $\sum_{i=1}^{n} c_{l} a_{l}$ where $a_{1}, \ldots, a_{n}$ are distinct elements of $A$ and $c_{1}, \ldots, c_{n}$ are elements of the fixed finite set $C \cup\{0\}$, it would be possible to keep $A^{*}$ 4-thin because $C \cup\{0\}$ does not contain an arithmetic progression of length 4 . (Actually, because of the 
way the priorities are about to be arranged, $A^{*}$ will only be $k_{0}$-thin, where $k_{0}$ is obtained by recursive approximations.)

Keeping $A^{*} k_{0}$-thin is a new negative requirement $N_{0}$. The negative requirements $N_{-1}$ and $N_{0}$ together will keep a certain set of numbers out of $A$; clearly, we must introduce a new negative requirement $N_{1}$ that this set be $k_{1}$-thin for a suitable number $k_{1}$. Continuing inductively, we obtain negative requirements $N_{0}, N_{1}, \ldots$ We rank the requirements with priorities as follows: $N_{-1}, P_{0}, N_{0}, P_{1}, N_{1}, \ldots$

The strategy for each positive requirement $P_{e}$ is the obvious one:

if $A^{s} \cap W_{e}^{s}=\varnothing$ and $W_{e}^{s}$ contains an element $x$ not restrained from $A$ at $s$ by any $N_{i}, i<e, e$ is the least such, put the least such $x$ into $A$ at $s$.

If the negative requirement $N_{e}$ succeeds in keeping the set of numbers held out of $A$ by the requirements $N_{i}$ with $i<e k_{e}$-thin, and if $W_{e}$ is thick, then $P_{e}$ will eventually receive attention and be satisfied permanently.

In order to form a strategy for the negative requirements, we need some preliminary notions. In terms of the operator $\Phi_{C}$ of $\S 2$, if $C_{0}=$ $\left\{-1,0, \frac{1}{2}, 2\right\}$, then $A^{*} \subseteq \Phi_{C_{0}}(A)$. Next, in order to keep track of what is kept out of $A$, we shall inductively define finite sets $C_{0} \subseteq C_{1} \subseteq \cdots$ so that every number kept out of $A$ by any $N_{i},-1 \leq i<e$, is in $\Phi_{C_{e}}(A)$. Then $N_{e}$ will require $\Phi_{C_{e}}(A)$ to be $k_{e}$-thin for a certain number $k_{e}$. For any operator $\Psi: \mathscr{P}(\mathbf{Q}) \rightarrow \mathscr{P}(\mathbf{Q})$ and any $k \in \omega$, let

$$
\begin{aligned}
& \Psi^{(k)}(B)=\{q \in \mathbf{Q} \mid \Psi(B) \text { is } k \text {-thin } \\
& \& \Psi(B \cup\{q\}) \text { is not } k \text {-thin }\} \text {. }
\end{aligned}
$$

The following lemma will be used to obtain $C_{e+1}$ from $C_{e}$ effectively.

Lemma 9. Let $B, C \subseteq \mathbf{Q}$. There is a recursive function $F: \mathscr{P}_{\text {fin }}(\mathbf{Q}) \rightarrow$ $\mathscr{P}_{\text {fin }}(\mathbf{Q})$ such that if $C$ is finite, $k$-thin and $0 \in C$, then $\Phi_{C}^{(k)}(B) \subseteq \Phi_{F(C)}(B)$.

Proof. Let $C$ be a given $k$-thin finite set of rational numbers containing 0. Suppose $q \in \Phi_{C}^{(k)}(B)$; hence $\Phi_{C}(B)$ is $k$-thin, but $\Phi_{C}(B \cup\{q\})$ is not $k$-thin. We now carry out some elementary computations to express $q$ as a linear combination of elements of $B$ with the coefficients from a finite set $F(C)$ not dependent on $q$ or $B$. Let $r_{1}, \ldots, r_{k}$ be a (nonconstant) arithmetic progression of length $k$ in $\Phi_{C}(B \cup\{q\})$. Hence each $r_{i}$ may be written as

$$
r_{i}=b_{i}+c_{i} q \quad \text { where } b_{i} \in \Phi_{C}(B) \text { and } c_{i} \in C
$$


(Note that here the fact that $0 \in C$ allows us to cover the case that $r_{i} \in \Phi_{C}(B)$; also note that $b_{i}$ 's are not necessarily distinct.) Since $r_{1}, \ldots, r_{k}$ is an arithmetic progression and $\Delta^{2}$ is linear, we have

$$
0=\Delta^{2} r_{i}=\Delta^{2} b_{i}+q \Delta^{2} c_{i} \text { for } 1 \leq i<k-1 .
$$

First assume for the sake of a contradiction that $\Delta^{2} c_{i}=0$ for all $i$, $1 \leq i<k-1$. Then $c_{1}=c_{2}=\cdots=c_{k}$ since otherwise $c_{1}, \ldots, c_{k}$ would be an arithmetic progression of length $k$ in $C$. Next, since $r_{1}, \ldots, r_{k}$ is not a constant sequence, neither is $b_{1}, \ldots, b_{k}$. But $\Delta^{2} b_{i}=0$ for $1 \leq i<k-1$ from (1) and by our hypothesis that $\Delta^{2} c_{i}=0$. Therefore $b_{1}, \ldots, b_{k}$ is an arithmetic progression of length $k$ in $\Phi_{C}(B)$, which is the desired contradiction.

Fix $i, 1 \leq i<q-1$, so that $\Delta^{2} c_{i} \neq 0$. From (1) we have

$$
q=-\Delta^{2} b_{l} / \Delta^{2} c_{l} \text {. }
$$

Since $b_{j} \in \Phi_{C}(B), i \leq j \leq i+2$, there are distinct numbers $a_{1}, \ldots, a_{n} \in B$ such that $b_{J}=\sum_{t=1}^{n} c_{j, t} a_{t}$ for $i \leq j \leq i+2$ and certain numbers $c_{j, t} \in C$. (Here, we use the fact that $0 \in C$ to make $a_{1}, \ldots, a_{n}$ independent of $j$.) By linearity of $\Delta^{2}$,

$$
\Delta^{2} b_{l}=\sum_{t=1}^{n} a_{t} \Delta^{2} c_{i t}
$$

where

$$
\Delta^{2} c_{i, t}=c_{i+2, t}-2 c_{t+1, t}+c_{i, t} .
$$

Since we are in search of $F(C)$ in order to have $q \in \Phi_{C}^{(k)}(B) \subseteq \Phi_{F(C)}(B)$, (2), (3), and (4) suggest that we may take $F(C)$ to be the set of all numbers of the form $-u / v$ where $v \neq 0$ and $u, v$ are each of the form $c-2 d+e$ with $c, d, e \in C$.

Let

$$
C_{0}=\left\{-1,0, \frac{1}{2}, 2\right\} \quad \text { and } \quad C_{e+1}=C_{e} \cup F\left(C_{e}\right)
$$

where $F$ is the function from Lemma 9. We enumerate $A$ in stages by defining $A^{s}$ for any $s$. At the end of stage $s$ we define $k_{e}^{s}$ so that $C_{e}$ and $\Phi_{C_{e}}\left(A^{s}\right)$ are $k_{e}^{s}$-thin. During the construction we need to satisfy the positive requirements $P_{e}$.

Definition. $P_{e}$ requires attention at stage $s+1$ if there exists $x$ such that

$$
A^{s} \cap W_{e}^{s}=\varnothing,
$$




$$
x \in W_{e}^{s}-\Phi_{C_{e}}\left(A^{s}\right) .
$$

Construction.

Stage 0. To initialize, let

$$
\begin{aligned}
& A^{0}=\varnothing, \text { and, for each } e, \\
& k_{e}^{0}=\text { the least } k \text { such that } C_{e} \text { is } k \text {-thin, }
\end{aligned}
$$

Stage $s+1$. If no $P_{e}$ requires attention, let

$$
A^{s+1}=A^{s} \quad \text { and } \quad k_{e}^{s+1}=k_{e}^{s} \text { for all } e
$$

and go on to the next stage. Otherwise, let $j$ be the least $e$ such that $P_{e}$ requires attention and choose the least corresponding $x$. Let

$$
A^{s+1}=A^{s} \cup\{x\}
$$

and

$$
k_{e}^{s+1}=\left\{\begin{array}{l}
k_{e}^{s} \quad \text { if } e<j \\
\text { the least } k \text { such that } \\
\quad C_{e} \text { and } \Phi_{C_{e}}\left(A^{s+1}\right) \text { are } k \text {-thin if } j \leq e .
\end{array}\right.
$$

(Note that the $k$ in the second case exists because $C_{e}$ and $\Phi_{C_{e}}\left(A^{s+1}\right)$ are finite sets.)

Since $A$ is clearly r.e., if the requirements $N_{-1}, P_{0}, N_{0}, \ldots$ are satisfied, the theorem is established. In the following we state and prove some lemmas which show that the requirements are indeed satisfied.

LEMMA 10. $A$ is 3-thin.

Proof. Note that

$$
\left(A^{s}\right)^{*} \subseteq \Phi_{C_{0}}\left(A^{s}\right) \subseteq \Phi_{C_{e}}\left(A^{s}\right)
$$

for all $e$ and $s$, so no element of $\left(A^{s}\right)^{*}$ enters $A$ at $s$ and $\left|A^{s+1}-A^{s}\right| \leq 1$. Hence as remarked before the constructions, it follows that $A$ is 3-thin.

LEMMA 11. For each e, $\lim _{s} k_{e}^{s}$ exists.

Proof. If $k_{e}^{s} \neq k_{e}^{s+1}$, then some $P_{J}$ with $j \leq e$ must have needed attention at stage $s+1$. But each $P_{j}$ receives attention at most once. 


\section{LEMMA 12. For each e and $s, C_{e}$ and $\Phi_{C_{e}}\left(A^{s}\right)$ are $k_{e}^{s}$-thin.}

Proof. This is proved by induction on $s$. It is clear if $s=0$. Suppose $C_{e}$ and $\Phi_{C_{e}}\left(A^{s}\right)$ are $k_{e}^{s}$-thin. Consider stage $s+1$; if no $P_{e}$ requires attention then the result for $s+1$ is immediate. Otherwise, assume $P_{j}$ requires attention at stage $s+1$ via $x$. By construction, $x$ is the unique element of $A^{s+1}-A^{s}$. If $e \geq j$, then $C_{e}$ and $\Phi_{C_{e}}\left(A^{s+1}\right)$ are $k_{e}^{s+1}$-thin by the choice of $k_{e}^{s+1}$. Suppose $e<j$. For the sake of a contradiction, assume that $\Phi_{C_{e}}\left(A^{s+1}\right)$ is not $k_{e}^{s+1}$-thin, and hence not $k_{e}^{s}$-thin. Therefore $x \in$ $\Phi_{C_{e}}^{\left(k_{e}^{s}\right)}\left(A^{s}\right)$. Since $\Phi_{C_{e}}\left(A^{s}\right)$ and $C_{e}$ are $k_{e}^{s}$-thin, it follows by choice of $C_{e+1}$ that $x \in \Phi_{C_{e+1}}\left(A^{s}\right)$. But $e+1 \leq j$, so $C_{e+1} \subseteq C_{j}$ and hence $x \in \Phi_{C_{j}}\left(A^{s}\right)$, contrary to the construction.

LEMMA 13. If $W_{e}$ is thick, then $A \cap W_{e} \neq \varnothing$.

Proof. Let $k_{e}=\lim _{s} k_{e}^{s}$. From Lemma 12 and the monotonicity and continuity of $\Phi_{C_{e}}$, it follows that $\Phi_{C_{e}}(A)$ is $k_{e}$-thin. Assume $e$ is such that $W_{e}$ is thick and $A \cap W_{e}=\varnothing$. Then $W_{e} \nsubseteq \Phi_{C_{e}}(A)$, so choose $x \in W_{e}-$ $\Phi_{C_{e}}(A)$. By monotonicity, $x \in W_{e}^{s}-\Phi_{C_{e}}\left(A^{s}\right)$ for all sufficiently large $s$, so $P_{e}$ will require attention at all sufficiently large stages and will eventually receive it, since each $P_{i}, i<e$, receives attention at most once.

This completes the proof of the theorem.

5. A corollary. To see a corollary consider:

Definition 14. Let $B \subseteq \omega . B$ is said to have positive upper density if

$$
\lim _{n \rightarrow \infty} \sup \frac{1}{n}|B \cap\{1, \ldots, n\}|>0 \text {. }
$$

It can be seen that a thick set does not have to have positive upper density. However, Erdös-Turán conjectured a phenomenon, later proved intricately by Szemerédi [3], one of whose consequences is that every set of positive upper density is thick.

Now, clearly the van der Waerden Theorem is a special case of Szemerédi's theorem since if a set $B$ is $k$-thin for any $k$ then it is not thick and therefore not of positive upper density. Hence it can be seen that $\omega-B$ has to be of positive upper density and hence it must have a thick subset.

Theorem 7 and Szemerédi's theorem may be used to give a counterexample to effectiveness of the Szemerédi's theorem. 
COROllaRy 15. There exists an r.e. set $A$ which is thin but intersects every r.e. set of positive upper density.

6. Generalization. It may be the case that Theorem 7 can be generalized in different ways. In this section we give one version which has a proof structurally the same as our proof of Theorem 7 .

Definition 16. Let $A \subset \omega$ and $k, d \in \omega . A$ is $k$-thin for degree $d$ if there is no nonconstant polynomial $p(x)$, with coefficients in $\mathbf{Q}$ and of degree $\leq d$ with $p(0), \ldots, p(k-1) \in A$.

Before we give the theorem, we state some useful facts. Clearly $A$ is $k$-thin for degree 1 iff $A$ is $k$-thin. Also, if $A$ is $k$-thin for degree $d$ and $A$ has more than 1 element, then $k \geq d+2$. This is because Lagrange's Interpolation Formula applied to given pairs $\left(x_{0}, y_{0}\right), \ldots,\left(x_{d}, y_{d}\right)$ with distinct $x_{0}, \ldots, x_{d}$ produces a polynomial $p$ of degree $\leq d$ such that $p\left(x_{i}\right)=y_{i}$ for $0 \leq i \leq d$.

Definition 17. $A$ is sparse for degree $d$ if for all $d^{\prime} \leq d, 0<d^{\prime}, A$ is $\left(d^{\prime}+2\right)$-thin for degree $d^{\prime}$. Note that $A$ is sparse for degree 1 iff $A$ is 3-thin, and if $A$ is sparse for degree $d$ and $d^{\prime} \leq d$, then $A$ is sparse for degree $d^{\prime}$.

Definition 18. $A$ is thick for degree $d$ if $A$ is not $k$-thin of degree $d$ for any $k$.

Finally we are able to generalize Theorem 7 whose intuitive content is 'there are sparse r.e. sets which meet every thick r.e. set'.

THEOREM 19. For each $d$, there is an r.e. set $A$ which is sparse for degree $d$ and intersects every r.e. set which is thick for degree $d$.

We leave the proof to the reader with a hint: in proof for Theorem 7, replace $\Delta^{2}$ by $\Delta^{d+1}$ and modify $C_{0}$ appropriately.

Observe that Theorem 19 reduces to Theorem 7 when $d=1$ and that the strength of Theorem 19 increases as $d$ increases.

7. Open questions. Upon further reflection, it becomes clear that Theorems 5 and 7 are about "controlling" thinness. This raises the following questions:

(1) Is there a recursive function $f$ and a 3-thin r.e. set $A$ such that $A \cap W_{e} \neq \varnothing$ whenever $W_{e}$ is $f(e)$-thick? By Theorem $5, f$ may not be 
chosen to be constant. By Theorem 7, there is such an $f$ recursive in $0^{\prime}$, i.e. $f(e)=k_{e}$.

In Theorem 19 we stated a generalized form of Theorem 7 . In that light

(2) Is there an r.e. set $A$ which is sparse for all degrees $d$ and which meets every r.e. set which is thick for any degree $d$ ?

Acknowledgement. We wish to thank Michael Ingrassia and Jeff Remmel for helpful conversations.

\section{REFERENCES}

[1] R. Graham, B. Rothchild and J. Spencer, Ramsey Theory, Wiley, 1980.

[2] A. Y. Khinchin, Three Pearls of Number Theory, Graylock Press, Rochester, New York 1952.

[3] H. Rogers, Jr., Theory of Recursive Functions and Effective Computability, McGrawHill, New York 1967.

[4] E. Szemerédi, On sets of integers containing no $k$ elements in arithmetic progressions, Acta Arith., 27 (1975), 299-345.

[5] B. van der Waerden, Beweis einer Baudetschen Vermutung, Nieuw Arch. Wisk, 15 (1927), 212-216.

[6] How the Proof of Baudet's Conjecture Was Found, Studies in Pure Mathematics (edited by L. Mirsky), Academic Press, pp. 251-260 (1971).

Received December 21, 1982. Research of the first author was partially supported by the National Science Foundation.

UNIVERSITY OF ILLINOIS

URBANA, IL 61801

AND

WESTERN ILLINOIS UNIVERSITY

MACOMB, IL 61455 



\title{
PACIFIC JOURNAL OF MATHEMATICS \\ EDITORS
}

\author{
DoNALD BABBITT (Managing Editor) \\ University of California \\ Los Angeles, CA 90024 \\ J. DugunduI \\ University of Southern California \\ Los Angeles, CA 90089-1113 \\ R. FINN \\ Stanford University \\ Stanford, CA 94305 \\ HERMANN FlasChKa \\ University of Arizona \\ Tucson, AZ 85721
}

C. C. Moore

University of California

Berkeley, CA 94720

ARTHUR OGUS

University of California

Berkeley, CA 94720

Hugo Rossi

University of Utah

Salt Lake City, UT 84112

H. SAMELSON

Stanford University

Stanford, CA 94305

ASSOCIATE EDITORS
R. ARENS
E. F. BECKENBACH
B. H. NeUMANN
F. WOLF
K. YoSHIDA (1906-1982)

\section{SUPPORTING INSTITUTIONS}

UNIVERSITY OF ARIZONA
UNIVERSITY OF BRITISH COLUMBIA
CALIFORNIA INSTITUTE OF TECHNOLOGY
UNIVERSITY OF CALIFORNIA
MONTANA STATE UNIVERSITY
UNIVERSITY OF NEVADA, RENO
NEW MEXICO STATE UNIVERSITY
OREGON STATE UNIVERSITY
UNIVERSITY OF ARIZONA
CALIFORNIA INSTITUTE OF TECHNOLOGY
UNIVERSITY OF CALIFORNIA
UNIVERSITY OF NEVADA, RENO
OREGON STATE UNIVERSITY
UNIVERSITY OF OREGON
UNIVERSITY OF SOUTHERN CALIFORNIA
STANFORD UNIVERSITY
UNIVERSITY OF HAWAII
UNIVERSITY OF TOKYO
UNIVERSITY OF UTAH
WASHINGTON STATE UNIVERSITY
UNIVERSITY OF WASHINGTON 


\section{Pacific Journal of Mathematics}

Vol. 115, No. $1 \quad$ September, 1984

Carlos Andradas Heranz and José Manuel Gamboa Mutuberría, A note on projections of real algebraic varieties $\ldots \ldots \ldots \ldots \ldots \ldots \ldots \ldots \ldots \ldots$

Jürgen Appell and Maria Patrizia Pera, Noncompactness principles in nonlinear operator approximation theory $\ldots \ldots \ldots \ldots \ldots \ldots \ldots \ldots \ldots \ldots$

Timothy John Carlson, Extending Lebesgue measure by infinitely many

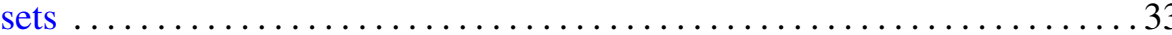

Donald S. Coram and Paul Frazier Duvall, Jr., Non-cell-like

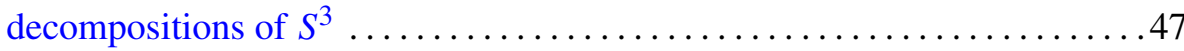

Edward Norman Dancer, Order intervals of selfadjoint linear operators and

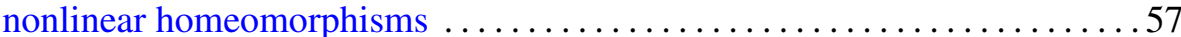

Ralph Jay De Laubenfels, Well-behaved derivations on $C[0,1] \ldots \ldots \ldots 73$

D. Feyel and A. de La Pradelle, Sur certaines extensions du théorème

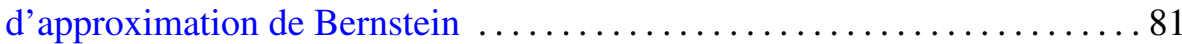

Colin C. Graham and Bertram Manuel Schreiber, Bimeasure algebras on

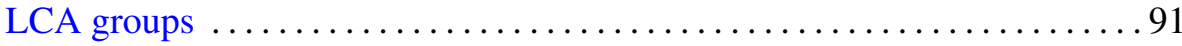

Richard Howard Hudson, Class numbers of imaginary cyclic quartic fields

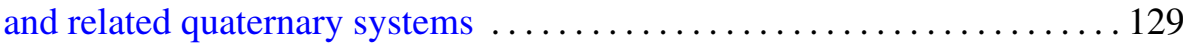

Carl Groos Jockusch, Jr. and Iraj Kalantari, Recursively enumerable sets and van der Waerden's theorem on arithmetic progressions . . . . . . . . 143

J. F. McClendon, On noncontractible valued multifunctions ........... 155 Akihiko Miyachi, Weak factorization of distributions in $H^{p}$ spaces $\ldots \ldots \ldots 165$ Ezzat S. Noussair and Charles Andrew Swanson, Global positive solutions of semilinear elliptic problems

Jon Christopher Snader, Strongly analytic subspaces and strongly

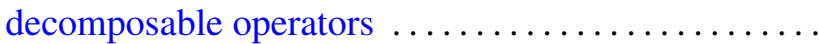

Boguslaw Tomaszewski, A construction of inner maps preserving the Haar measure on spheres

Akihito Uchiyama, The Fefferman-Stein decomposition of smooth functions and its application to $H^{p}\left(\mathbf{R}^{n}\right) \ldots \ldots \ldots$ 